\title{
2014 Cole Prize in Number Theory
}

YitANG ZhANG, DANIEL GoldSTON, JÁNOS PINTZ, and CEM Y. YILDIRIM were awarded the 2014 Cole Prizes in Number Theory at the 120th Annual Meeting of the AMS in Baltimore, Maryland, in January 2014.

\section{Citation}

The 2014 Frank Nelson Cole Prize in Number Theory is awarded to Yitang Zhang for his work on bounded gaps between primes, and to Daniel

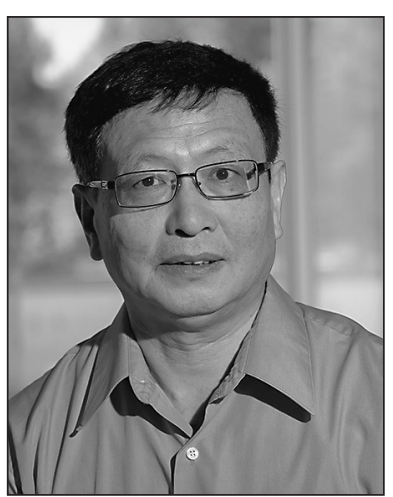

Yitang Zhang

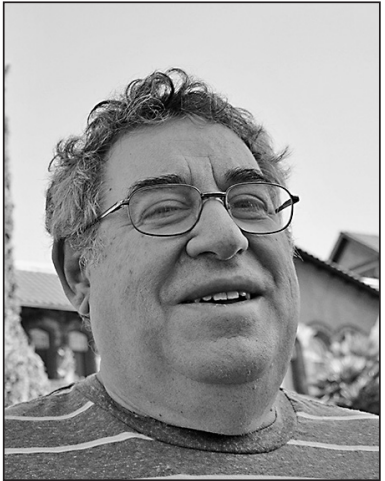

Daniel Goldston
Goldston, János Pintz, and Cem Y. Yıldırım for their work on small gaps between primes. This work appeared in two papers: Yitang Zhang, "Bounded gaps between primes", Annals of Mathematics (2) (to appear); and Daniel Goldston, János Pintz, and Cem Y. Yıldırım, "Primes in tuples. I", Annals of Mathematics (2) 170 (2009), no. 2, 819-862.

One of the oldest problems in number theory is the twin prime conjecture: that there are infinitely many pairs $p$ and $q$ of primes with $p-q=2$. With the Goldbach conjecture, this is an archetypal problem that motivated the development of sieve theory by Brun, Linnik, Chen, Selberg, Bombieri, Iwaniec, Friedlander, Heath-Brown, and many others. Yet the twin prime conjecture seemed far out of reach when in 2005, Goldston, Pintz, and Ylldırım (GPY) showed that for every $\epsilon>0$, there exist infinitely many pairs $p$ and $q$ of distinct primes with $|p-q|<$ $\epsilon \log (p)$. Their very surprising proof of this breakthrough result used standard tools-the Selberg

DOI: http://dx.doi.org/10.1090/noti1110 sieve and the Bombieri-Vinogradov theorem-together with innovative new ideas that are essentially combinatorial. The Bombieri-Vinogradov theorem is an error term for the prime number theorem for primes in an arithmetic progression. It is often used as a substitute for the generalized Riemann hypothesis in arithmetic applications. The Selberg sieve is a flexible tool, and the authors found a new and ingenious way of applying it to obtain their result. Slightly earlier work of Goldston and Ylldırım on this idea played a role in the proof by Green and Tao that primes exist in arbitrarily long arithmetic progressions.

After GPY there was optimism that improvements in the Bombieri-Vinogradov theorem might yield bounded gaps between primes. Such improvements had already been found by Iwaniec, Fouvry, and Friedlander, culminating in the 1989 result of Bombieri, Friedlander, and Iwaniec. This result is an error term for the prime number theorem in a family of arithmetic progressions $m x+a$ where $a$ is fixed and $m$ varies.

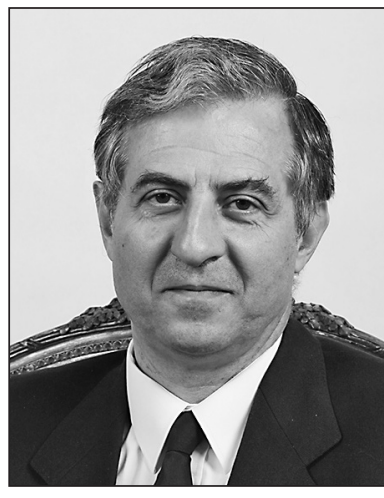

János Pintz

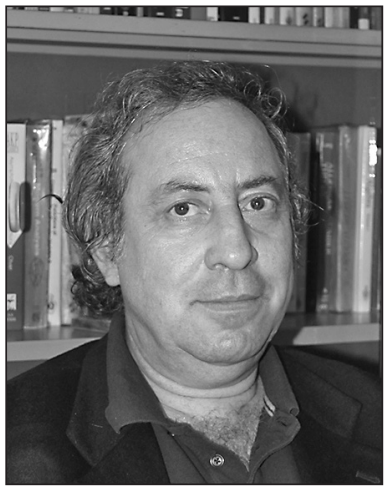

Cem Y. Yıldırım
Yet within a year or two of GPY, no one saw how to use the results of Bombieri, Friedlander, and Iwaniec to prove bounded gaps between primes. So the initial optimism gave way to pessimism. But then, very unexpectedly, Yitang Zhang did find a way, and he proved the striking result that there are infinitely many pairs $p, q$ of distinct primes with $|p-q|<7 \times 10^{7}$. Zhang saw that a different modification of the Bombieri-Vinogradov theorem could be used, in which the modulus $m$ is constrained, together with a modification of the sieve ideas of Goldston, Pintz, and Ylldırım. Beyond the crucial initial insight, carrying out this plan combines input from several of number theory's most illustrious ideas. For example, the Riemann hypothesis for curves, due to Weil, and that for varieties, due to Deligne, are essential parts for his argument, as is the dispersion method of Linnik, which is used to transport the sieve inequality to the relevant exponential sum bounds. 


\section{Biographical Sketch: Yitang Zhang}

Yitang Zhang received his B.S. from Peking University and his Ph.D. from Purdue University. Since 1999, he has been a lecturer in the Department of Mathematics and Statistics of the University of New Hampshire. His research is mainly in the field of analytic number theory, in particular the distribution of prime numbers and the distribution of zeros of the zeta function. He received the 2013 Morningside Special Achievement Award in Mathematics from the International Conference of Chinese Mathematicians in July 2013. Recently, he was appointed a full professor by the University of New Hampshire.

\section{Response from Yitang Zhang}

I am humbled and honored to have been selected as the corecipient of the Frank Nelson Cole Prize in Number Theory. This honor should really be credited to those who have influenced my work. In particular, it is the work of Bombieri, Fouvry, Friedlander, and Iwaniec on the stronger versions of the BombieriVinogradov theorem that provides indispensable tools for bounding the error terms. I am grateful to the Annals of Mathematics for the quick reaction on my submission and to the referees for studying the manuscript thoroughly and making useful comments; I had not expected that the paper would be accepted within a few weeks. Finally, I must thank the people who contributed great help in my academic career: Dr. Perry Tang, Professor Liming Ge, and the late Professor Kenneth Appel.

\section{Biographical Sketch: Daniel Goldston}

Daniel Goldston was born on January 4, 1954, in Oakland, California. He attended the University of California Berkeley starting in 1972, receiving his Ph.D. in 1981 under the supervision of R. Sherman Lehman. He worked at the University of Minnesota Duluth for a year before spending the 1982-1983 academic year at the Institute for Advanced Study in Princeton. Since 1983 he has worked at San Jose State University except for semesters spent at the Institute for Advanced Study in 1990, the University of Toronto in 1994, and the Mathematical Sciences Research Institute in 1999.

\section{Response from Daniel Goldston}

The mathematical work for which this prize has been awarded was aided by many mathematicians over many years, but I will not attempt to thank them all individually. Let me tell a story which starts in 1999, when, for many of us, the recent stunning work of Yitang Zhang would have seemed less likely than a proof of the Riemann Hypothesis.

Cem and I were both visiting MSRI in Berkeley, and one day he came in and said that rather than working on the paper we were supposed to be writing, he had been trying to work out a triple correlation divisor sum. We started working on this together and, after a few weeks, began to see that it was possible to work out asymptotic formulas for this type of sum. Over the rest of the term we continued to work on this, at first getting different answers each time we did the calculation but eventually tending toward only one answer. Cem went back to Turkey, but we continued our joint work by email, slowly working out asymptotic formulas for these sums. This was incremental research, the only kind I actually know how to do, where we used standard classical methods and neither knew nor expected any exciting applications. At the time with little kids of ages 0,2 , and 4 in the house, and fragmented times for work, doing these calculations was ideal since they could be interrupted and then easily resumed. Finally, when the kids were 3, 5, and 7 in 2003, Cem and I thought we had made a breakthrough on gaps between primes but, while we received a lot of publicity, this did not help change the fact that the proof was wrong. Math can be a tough business, and while mathematicians often do not have much humility, we all have lots of experience with humiliation. Fortunately, in this case our work was not destined for the wastebasket, and in 2004 Green and Tao found a use for our formulas in their work on arithmetic progressions of primes, and in 2005, with János Pintz, we obtained the GPY method which proved new results on gaps between primes and provided part of the foundation for Zhang's great advance in 2013.

\section{Biographical Sketch: János Pintz}

János Pintz received his M.Sc. at the Eötvös Loránd University in Budapest, Hungary, in 1974 and his Ph.D. (so-called candidate's degree) from the Hungarian Academy of Sciences in 1975 under the supervision of Professor Paul Turán. After working for a few years at Eötvös Loránd University, since 1977 he has been a research fellow at the Mathematical Institute of the Hungarian Academy of Sciences, which today is called Alfréd Rényi Mathematical Institute of the Hungarian Academy of Sciences after its founder and first director, Alfréd Rényi. During this period, Pintz was visiting professor at several foreign universities for a few years. His research focuses on prime number theory. In the past twenty years he worked mostly on three of the four famous problems mentioned by Landau more than a hundred years ago in his invited address at the International Congress of Mathematicians in Cambridge in 1912; namely, the Goldbach conjecture, the twin prime conjecture, and large gaps between consecutive primes. He is a member of the Hungarian Academy of Sciences and the Academy of Europe.

\section{Response from János Pintz}

I am grateful to the American Mathematical Society and the Selection Committee for the great honor of choosing me as one of the corecipients of the 2014 Frank Nelson Cole Prize in Number Theory. 
I have to say that when I first learned some three or four decades ago that my famous fellow citizen, Paul Erdós, received this award for his contribution to the elementary proof of the Prime Number Theorem, I could not have imagined that one day I would be honored with the same distinction. This is even more true if one takes a quick look at the list of giants in number theory who received this prize after Paul Erdôs in the past sixty years. First I would like to thank my late Professor Paul Turán, who showed me the beauty of primes in his lecture when I was still a first-year undergraduate student at Eötvös University, and who was my advisor until his unduly early death at age sixty-six in 1976. I also would like to thank my friends and colleagues Endre Szemerédi and Gábor Halász, from whom I learned very much in the later stage of my career. My special thanks are due to my coauthors, friends, and corecipients of this prize, Dan Goldston and Cem Ylldırım, for the very friendly and fruitful collaboration during the past years which led to a number of results about small gaps between primes. I also thank my wife and children, the Alfréd Rényi Mathematical Institute of the Hungarian Academy of Sciences, and finally God for a fortunate life, in that I was able to devote most of my time to my work, hobby, and obsession: to think about mathematical problems, especially those connected with the mysteries of primes. In this respect I can quote one of the greatest mathematicians of all times, Leonhard Euler, who once said, "Mathematicians have tried in vain to this day to discover some order in the sequence of prime numbers, and we have reason to believe that it is a mystery into which the human mind will never penetrate." I am very glad that standing on the shoulders of giants, such as Birch, Bombieri, Deligne, Fouvry, Friedlander, Heath-Brown, Iwaniec, Motohashi, Selberg, and Weil, we, the recipients of the 2014 Frank Nelson Cole Prize, could somewhat reduce the domain of mysteries of primes into which the human mind can never penetrate.

\section{Biographical Sketch: Cem Y. Yıldırım}

Cem Yalçın Yıldırım was born in Bloomington, Indiana, in 1961. He grew up in Ankara, Turkey, and received his B.Sc. degree in physics from Middle East Technical University (METU), Turkey, in 1982, and his Ph.D. from the University of Toronto, Canada, in 1990 under the supervision of John B. Friedlander. Since 2002, he is professor of mathematics at Boğaziçi University, Istanbul, Turkey, studying mostly analytic number theory and classical analysis.

\section{Response from Cem Y. Yıldırım}

I am humbled and honored to be one of the recipients of the 2014 Frank Nelson Cole Prize in Number Theory. My education began at home, where I was raised in an intellectually stimulating environment. I am grateful to my parents for always encouraging me to concentrate upon anything that interested me. I was completely enraptured when I stumbled upon the classic book on number theory by Hardy and Wright in the library of my high school, and from then on I knew what area I wanted to learn most. The Department of Mathematics at METU had an excellent atmosphere for an undergraduate eager to absorb mathematics.

I am grateful to the University of Toronto, where I was granted scholarship throughout the years I pursued my studies toward a dissertation, and to my Ph.D. thesis supervisor, John B. Friedlander, who always gave me his support even well after I graduated. In 1995 I began collaborating with Dan Goldston on his so-called lower bound method while on sabbatical at San Jose State University. Later on, visits to MSRI, MFO, and IAS were very beneficial to me. I am deeply indebted to Dan Goldston and János Pintz for always sharing ideas freely. Our collaboration has been a great learning process for me. I would also like to thank Boğaziçi University for providing enlightened and peaceful working and living conditions.

\section{About the Prize}

The Cole Prize in Number Theory is awarded every three years for a notable research memoir in number theory that has appeared during the previous five years. The awarding of this prize alternates with the awarding of the Cole Prize in Algebra, also given every three years.

These prizes were established in 1928 to honor Frank Nelson Cole (1861-1926) on the occasion of his retirement as secretary of the AMS after twenty-five years of service. He also served as editor-in-chief of the Bulletin for twenty-one years. The endowment was made by Cole and has received contributions from Society members and from Cole's son, Charles A. Cole. The Cole Prize carries a cash award of US\$5,000.

The Cole Prize in Number Theory is awarded by the AMS Council acting on the recommendation of a selection committee. For the 2014 prize, the members of the selection committee were Daniel Bump, B. H. Gross, and Audrey A. Terras.

Previous recipients of the Cole Prize in Number Theory are H. S. Vandiver (1931), Claude Chevalley (1941), H. B. Mann (1946), Paul Erdős (1951), John T. Tate (1956), Kenkichi Iwasawa (1962), Bernard M. Dwork (1962), James B. Ax and Simon B. Kochen (1967), Wolfgang M. Schmidt (1972), Goro Shimura (1977), Robert P. Langlands (1982), Barry Mazur (1982), Dorian M. Goldfeld (1987), Benedict H. Gross and Don B. Zagier (1987), Karl Rubin (1992), Paul Vojta (1992), Andrew J. Wiles (1997), Henryk Iwaniec (2002), Richard Taylor (2002), Peter Sarnak (2005), Manjul Bhargava (2008), and Chandrashekhar Khare and Jean-Pierre Wintenberger (2011).

-Elaine Kehoe 